\title{
A Control Strategy for Multi-Robot System Navigating in a Dynamic Environment
}

\author{
Ting Wang, Ryad Chellali, Yang Yi, Wen Qin, Mingzhu Wei \\ College of Electric Engineering and Control Science, Nanjing Tech University, Nanjing, China \\ Email: wangting0310@qq.com
}

Received October 2015

\begin{abstract}
This paper introduces a new control strategy for heterogeneous multi-robots systems dedicated to industrial logistic setups. This control strategy is based on both distributed intelligence and machine learning and involves three parts: the rigid formation controller, the perception system and the path planner. Our controller is event-based and thus its control-coordination strategy can be self-adaptive and applied to real dynamic environment. During the navigating process, the multi-robots system derives the environment model, performs the path planning process that guaranties both the transportation constraints and the obstacle avoidance. For the validation, both simulation and real robot experiments are performed. The results show that the developed control strategy can be well used for realistic logistics applications.
\end{abstract}

\section{Keywords}

Multi-Robots System, Distributed Intelligence, Machine Learning, Dynamic Environment

\section{Introduction}

Since 1980s, researcher interests in multi-robots systems (MRS) are increasing as it becomes clear that they offer more benefits compared single robot systems [1] [2]: MRS are more robust and they support higher mobility. Indeed, their inherent redundancy reduces the effects of individual malfunctions while having a high number of degrees of freedom for the full system. These advantages are counter-balanced by a higher complexity, i.e., one has more challenging control and path planning issues to solve. In addition to the classical control/oath planning problems, MRS should consider the interactions among robots' group under four umbrellas: collective, cooperative collaborative and coordinative behaviors [3]. For some applications, only part these behaviors are necessary. For the more general case, all of them are needed. In [4] for instance, an MRS is used for exploration task and the LUNARES (Lunar Exploration Scenario) system is used to gather information about its environment. In logistics applications for instance, all behaviors are necessary: the group of robots handles an object and move it for a given area to another area. In such cases, it is necessary to solve a multi-objective problem: 1) deriving the right formation (relative placement of robots) allowing transporting rigid objects, 2) performing accordingly path planning, including obstacles avoidance for individual robots.

In late 1980s, several projects about heterogeneous robotic systems have been presented. The first project was ACTRESS (Actor-based Robot and Equipment's Synthetic System) project [5]-[7], which is inspired by the 
Universal Modular ACTOR Formalism [8]. The second project was GOFFER [9] [10]. In GOFFER, a central task planning and scheduling system (CTPS) can communicate with all robots and can get a global view. Likewise, the ALLIANCE architecture was developed by Parker in [11] [12] to study the cooperation in a heterogeneous, small-to-medium-sized team of largely independent, loosely coupled robots.

As for stepping from one to more robots, involving heterogeneity in MRS has a cost: the design of the control strategy is harder due to the multiplicity of locomotion. Indeed controlling simultaneously a walking robot together with a wheeled robot to achieve a given formation requires combining two different state spaces for which solutions are not straightforward (moving a non-holonomic system and maintaining the equilibrium of a biped). To cope with these difficulties, we addressed the issue of unifying the four previous requirements to obtain a single formalism allowing solving a multi-objective optimization problem where both robots as a group and as individuals should obey to simultaneous constraints dealing with rigid formation and obstacle avoidance.

In this paper, we present an application related to logistics. Our goal is allowing the MRS transporting different kinds of objects from one point to another point in unknown and varying environments. The size, the shape and the weight of the transported objects may vary. As well, the working space is partially unknown and may vary itself. In this perspective, we used machine-learning techniques to make the MRS self-adaptive and reacting effectively and safely to any unexpected change in the working environment.

The contribution is organized as follows. Section 2 introduces the control strategy. Section 3 presents the rigid formation control of multi-robots system. Section 4 details the path planning algorithm as well as the perception system. Simulations and real robot experiments are given in Section 5. Conclusions and future work were given in the Section 6.

\section{System Overview}

\subsection{Task Description}

In real transportation, it usually needed to carry large and heavy loads in many situations, both in indoor and outdoor environments. In such cases, it is crucial to maintain the rigid formation of the multi-robots system in order to keep the load not falling down during the transport. In addition, the obstacle avoidance should be solved for individual robots as well as the multiple robots as a whole. Accordingly, the MRS should perform tasks as depicted in Figure 1 and Figure 2. It consists of maintaining rigid formation to carry objects. Meanwhile, the perception system establishes an occupation grid map allowing planning safe and feasible paths. The perception system is responsible for gathering the image and information of the current environment. In the paper, a camera or a flying robot materializes the perception system. With the occupancy grid and given the final destination, an optimal path is determined thanks to the Q-learning algorithm.

\subsection{Perception Module}

In order to get environment model, we developed a computer vision based module to extract changes. Our sys-

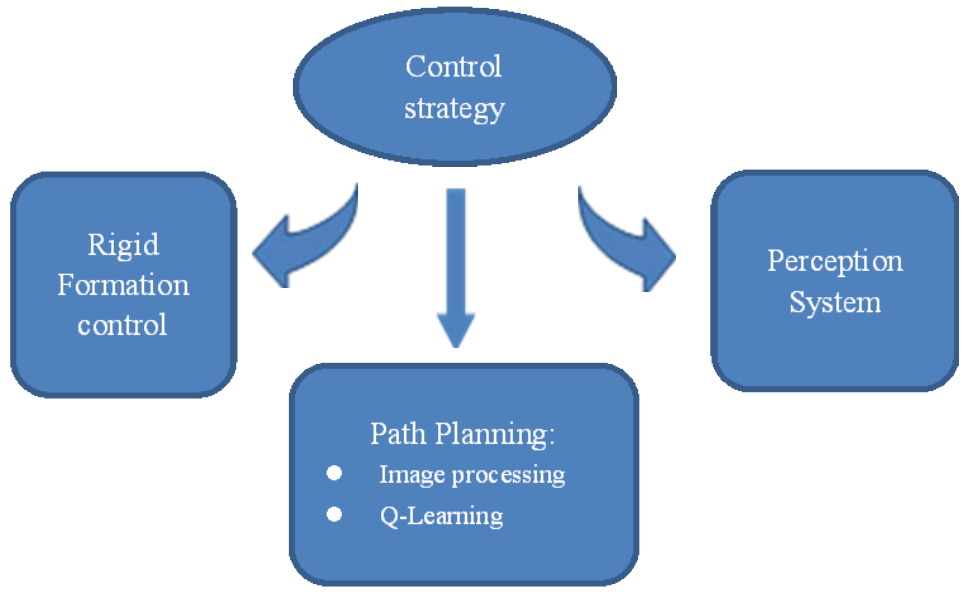

Figure 1. Schematic diagram of the control strategy. 


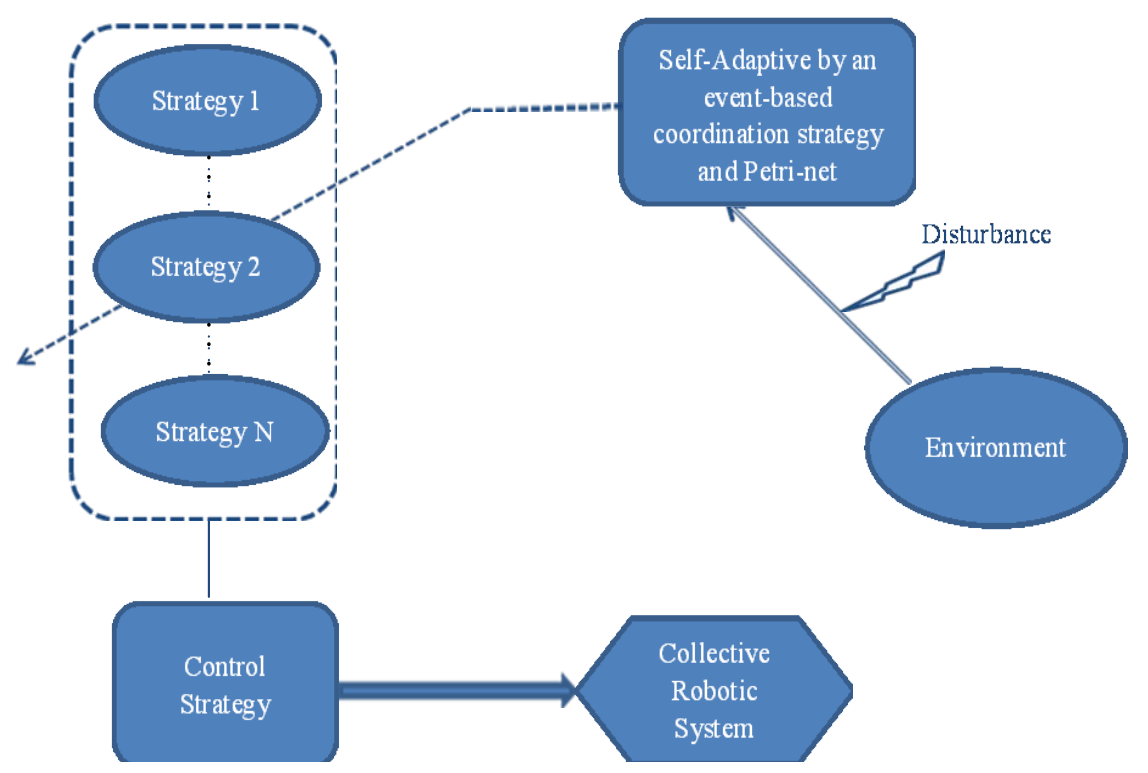

Figure 2. Even-based coordination strategy and Petri-net.

tem compares successive images taken by a UAV (or a moveable camera). After background substraction, we obtain a binary matrix $E(i, j)$.

$$
E=\left[\begin{array}{llllllllll}
1 & 0 & 1 & 1 & 1 & 1 & 1 & 1 & 1 & 1 \\
1 & 0 & 1 & 1 & 1 & 1 & 1 & 1 & 1 & 1 \\
1 & 0 & 0 & 0 & 0 & 0 & 1 & 0 & 0 & 0 \\
1 & 0 & 0 & 0 & 0 & 0 & 1 & 0 & 0 & 0 \\
1 & 1 & 1 & 1 & 0 & 0 & 1 & 0 & 0 & 0 \\
1 & 1 & 1 & 1 & 0 & 0 & 0 & 0 & 0 & 0 \\
1 & 0 & 0 & 0 & 0 & 0 & 0 & 0 & 0 & 1 \\
1 & 0 & 0 & 0 & 0 & 0 & 0 & 0 & 0 & 1 \\
1 & 0 & 0 & 0 & 0 & 0 & 0 & 0 & 0 & 1 \\
1 & 1 & 1 & 1 & 1 & 1 & 1 & 1 & 1 & 1
\end{array}\right]
$$

In which 1 represents obstacles and 0 is free path. Here, in order to adapt to our experimental robot (Khepera III), we reshape the size of matrix $E$ to fit to the real dimension of the working space (see in Figure 7). In this example, the environment is divided into 100 states where each state is a square of $30 \mathrm{~cm} \times 30 \mathrm{~cm}$. Rigid formation control part and Q-learning part can be found in [13]-[16].

\subsection{Example of Execution}

An example illustrated in Figure 3, it introduced how the control strategy works. It is a model of the virtual environment used in the simulation. The heterogeneous multi-robots team must cross a wide unknown area from A to B. The "unknown" means that they cannot get the complete environmental information at the initial time step. Since the area is huge and unknown, the heterogeneous multi-robots team must pass the area steps by steps. Supposed that, in each time, they cross an area $\left(3 \times 3 \mathrm{~m}^{2}\right)$ which we called a "room". In this virtual environment, the multi-robots system is composed of three Khepera III (a kind of two-wheel non-holonomic robot), a humanoid robot Nao (http://www.aldebaran-robotics.com/), a camera (role as perception system) and a virtual robot supervisor (data processing). The environment model is decomposed into 21 rooms (see Figure 3, the room is indicated by a two-dimension array, respectively along the axis-x and axis-y). Each room is divided into 100 states, and each state is a square with side of 30 centimeters. For example, the initial A locates in room $(2,2)$ and the final B locates in room $(1,7)$ (see Figure 4). The total area of the environment is equal to $6 \times 12 \mathrm{~m}^{2}$. 

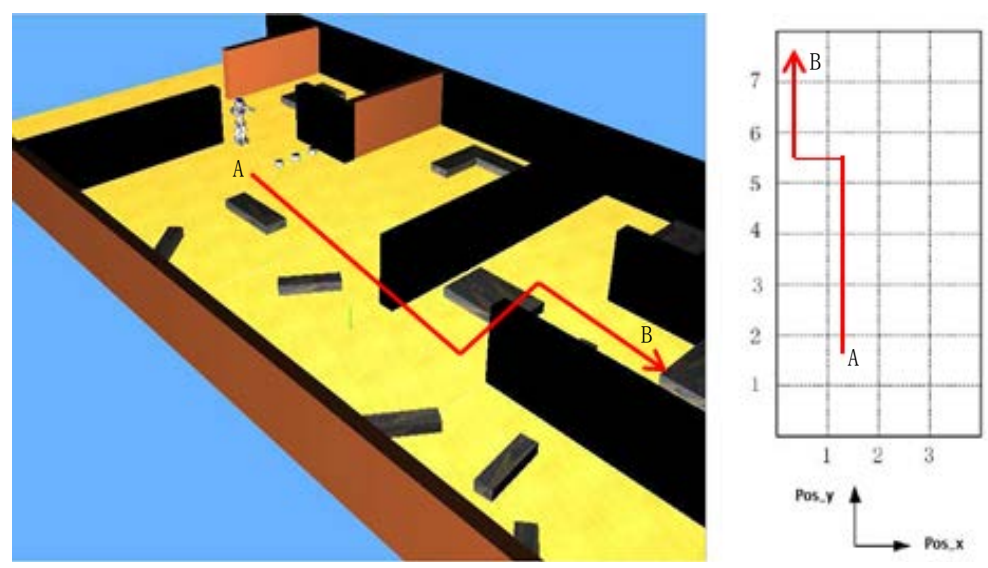

Figure 3. Virtual environment of the multi-robot system.

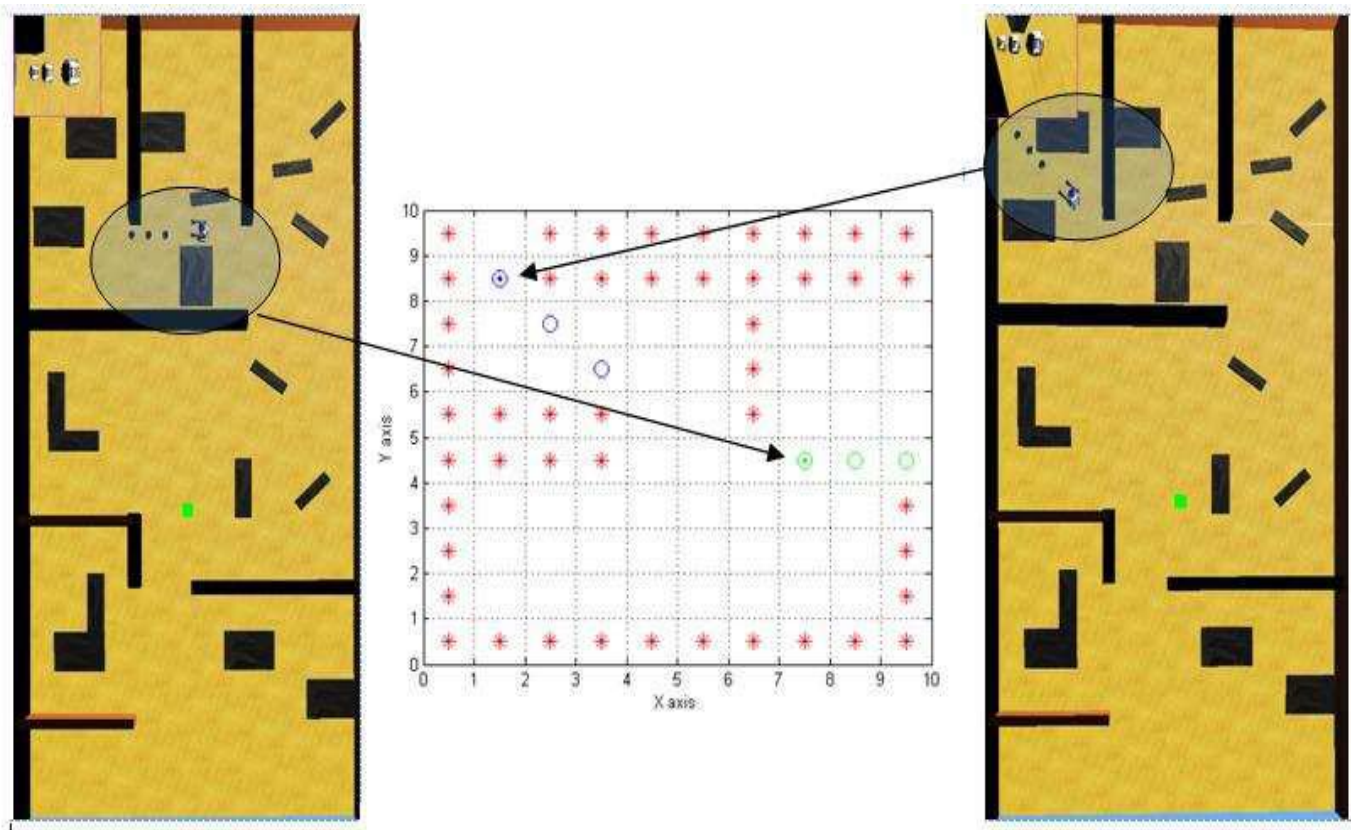

Figure 4. Illustration of the path planning task carried out by the top camera and remote computer: start point (left), goal point (right).

\section{Real Robot Experiment}

The real experiment consisted firstly to compute the path planning by the supervisor and secondly to control the rigid formation of multi-robots system. In the real experiment, we use two Khpera III robots, a humanoid robot Nao played a role of local supervisor, a camera and a computer acted as a supervisor for remote control of both camera and robots. The camera is hanging on the $2 \mathrm{~m}$ height. The real environment (in Figure 5(a)) constitutes by a $1.5 \mathrm{~m}$ wide and $2.1 \mathrm{~m}$ long rectangular floor. Some paper used to act as obstacles. The environment comprises 35 states and each state is a $30 \mathrm{~cm}$ side square.

\subsection{Environment and Path Planning}

The path planning of the unknown environment is displayed in Figure 5(b). The initial position (the green dot and circle) and final position (the blue dot and circle) respectively are [4; 2] and [1;6]. In the experiment, multirobots system maintained the vertical formation 2 from the beginning to the end. The initial position marked with green dot (the reference robot) and green circle. The final position marked with blue dot and blue circle. 


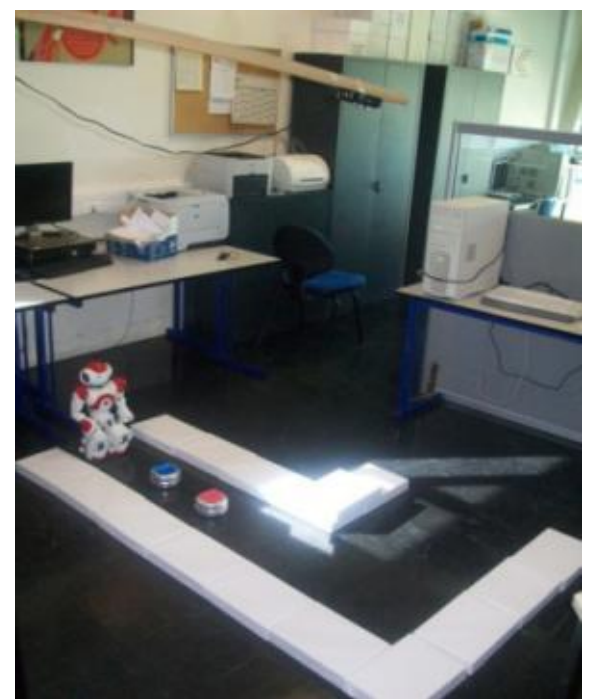

(a)

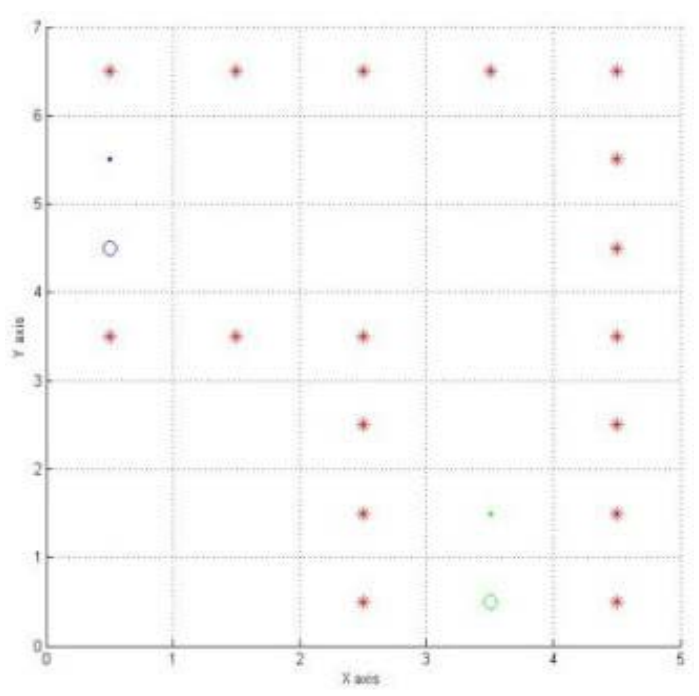

(b)

Figure 5. (a) Unknown environment; (b) Path planning results.

\subsection{Experiment Results}

Let us suppose that the multi-robots system entered the current room k (see in Figure 6(a)) from the previous room $k-1$. They aim to go across the room $\mathrm{k}$ and enter into next room $k+1$. The process includes two parts. In the first part, Nao led two Khepera III robots move ahead, because there is a free pass way in front of the multi-robots system on initial time step. In the second part, we put a barrier to block the pass way in front of the multi-robots system. Based on an event-based coordination strategy, HMRS can be self-adaptive to the sudden disturbance of the environment. The detailed process is as follows: (1) Nao explored the front area at each time step. As soon as it observed the obstacle, it enabled the top camera to send an image of current unknown environment. Then, through calculating the path planning by a supervisor, two Khepera III robots (multi-robots system) apply ANFIS rigid formation control to avoid obstacles. Nao executed the corresponding actions according to the results of path planning. Finally, multi-robots system crossed the current room $\mathrm{k}$ as well as they arrived at the initial position of room $k+1$. The results and description of go forwards part and path planning part are as follows.

The pictures of the first part displayed in Figure 6. At the beginning of the experiment, HMRS went into room $\mathrm{k}$ from room $k-1$. Nao led two Khepera III robots (MRS) keep going ahead as there is a pass way. The initial state of experiment showed in Figure 6(a) and the segment of the first part showed in Figure 6(b).

After the short go forwards part, we set an obstacle in order to stop HMRS and removed it after few seconds (see in Figure 7(a)). In this case, as Nao updated the current image and detected the obstacle, it demanded the external camera to give the image of the current room $\mathrm{K}$ to the supervisor. Using Q learning, the optimal path and actions obtained by supervisor (see in Figure 7(b)). Multi-robots System was interrupted and exchanged commands with the supervisor. The supervisor switched the go forwards control to rigid formation control.

The path planning demonstrated in Figure 7(b). Actions are 11333 for the rest part of environment. In the part, two Khepera III robots (MRS) formed a vertical line rigid formation. By the ANFIS rigid formation control, MRS crossed the area without colliding with obstacles. After two continuous go forwards translational actions (see in Figure 7(b) and Figure 7(c)), multi-robots system turned left to finish the rest three left translational actions. Nao followed the MRS to turn left (see in the Figure 7(d) and Figure 7(e)) after the two continuous forwards translational actions. The rest three actions illustrated respectively in Figures 7(d)-(f). The final state of the experiment showed in Figure 7(f). They will continue to move in the room $k+1$.

\section{Conclusion}

Comparing with existed control research of the heterogeneous multi-robots system, the paper proposed a control strategy based on the machine learning and an event-based coordination strategy. The obvious advantage of the 


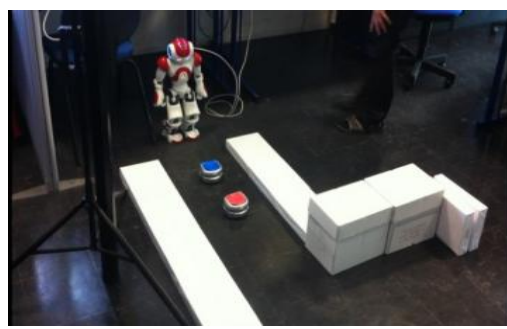

(a)

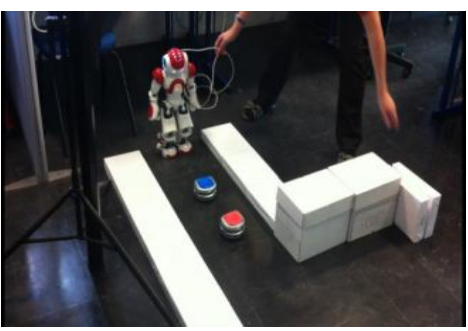

(b)

Figure 6. Go forwards part. (a) Initial state of the experiment; (b) Multi-robots team goes forwards.

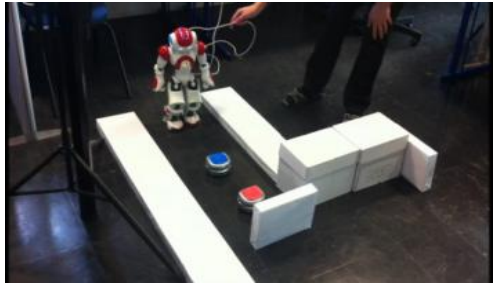

(a)

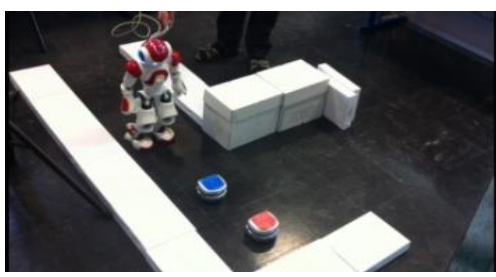

(c)

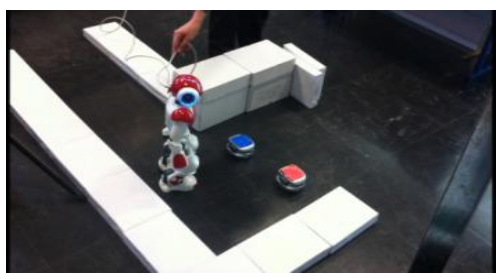

(e)

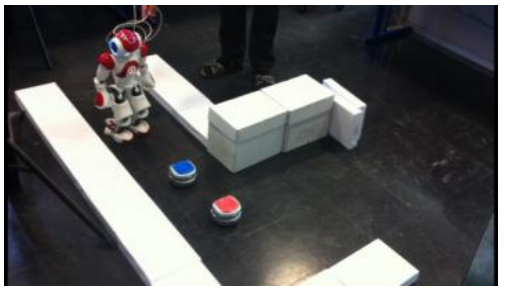

(b)

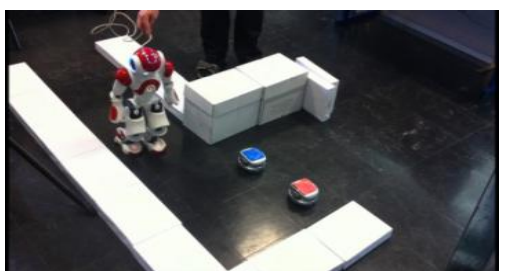

(d)

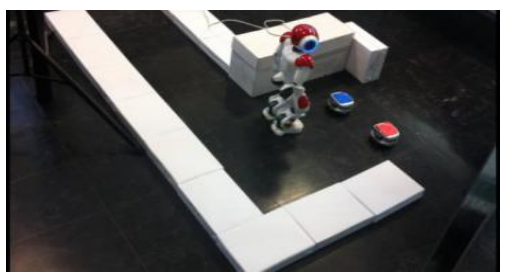

(f)

Figure 7. Path planning part.

strategy is that it makes the heterogeneous multi-robots team be self-adaptive to any disturbance of the current environment. Due to the coordination and cooperation among different types of robots, the HMRS can accomplish a common task by the unified distributed intelligence. Besides the simulation in a very widely virtual environment, the paper also validated the strategy with a real robots experiment. The strategy may be used to the practical logistic industry, such as environment exploring, transportation tasks and so on. In the future, the work will continue to focus on the adaptation of formation versus type of load aiming to apply in the logistic industry.

\section{References}

[1] Arai, T., Pagello, E. and Parker, L.E. (2002) Editorial: Advances in Multi-Robot Systems. IEEE Trans. Robot. Autom, 18, 655-661. http://dx.doi.org/10.1109/TRA.2002.806024

[2] Cao, Y., Fukunaga, A. and Kahng, A. (1997) Cooperative Mobile Robotics: Antecedents and Directions. Auton. Robot., 4, 1-23. http://dx.doi.org/10.1023/A:1008855018923

[3] Parker, L.E. (2008) Multiple Mobile Robot System. In: Bruno, S. and Oussama, K.S., Eds., Handbook of Robotics, Springer-Verlag, Berlin Heidelberg, 921-941. http://dx.doi.org/10.1007/978-3-540-30301-5_41

[4] Cordes, F., Ahrns, I., Bartsch, S., Birnschein, T., Dettmann, A., Estable, S., Haase, S., Hilljegerdes, J., Koebel, D., 
Planthaber, S., Roehr, T., Scheper, M. and Kirchner, F. (2010) LUNARES: Lunar Crater Exploration with Heterogeneous Multi Robot Systems. Intelligent Service Robotics, 6 December 2010, 1-29.

[5] Asama, H., Ishida, Y., Ozaki, K., Habib, M.K., Matsumoto, A., Kaetsu, H. and Endo, I. (1992) A Communication System between Multiple Robotic Agents. In: Leu, M., Ed., Proc. the Japan USA Symposium on Flexible Automation, 647-654.

[6] Alami, R., Fleury, S., Herrb, M., Ingrand, F. and Robert, F. (1998) Multi-Robot Cooperation in the MARTHA Project. Robot Automation. Mag, 5, 36-47.

[7] Ishida, Y., Endo, I. and Matsumoto, A. (1991) Communication and Cooperation in an Autonomous and Decentralized Robot System. IFAC Int. Symp. on Distributed Intelligent Systems, 299-304.

[8] Hewitt, C., Bishop, P., Greif, I., Smith, B., Matson, T. and Steiger, R. (1973) A Universal Modular Actor Formalism for Artificial Intelligence. Proc. Intl. Joint Conf. Artificial Intelligence, 235-245.

[9] Caloud, P., Choi, W., Latombe, J.C., Le Pape, C. and Yim, M. (1990) Indoor Automation with Many Mobile Robots. Proc. IEEE Int. Workshop Intell. Robot. Syst., Tsuchiura, 67-72. http://dx.doi.org/10.1109/iros.1990.262370

[10] LePape, C. (1990) A Combination of Centralized and Distributed Methods for Multi-Agent Planning and Scheduling. IEEE ICRA, 488-493.

[11] Parker, L.E. (1994) ALLIANCE: An Architecture for Fault Tolerant, Cooperative Control of Heterogeneous Mobile Robots. Proc. IEEE/RSJ/GI Int. Conf. Intell. Robot. Syst., Munich, 776-783. http://dx.doi.org/10.1109/IROS.1994.407550

[12] Parker, L.E. (1994) Heterogeneous Multi-Robot Cooperation. Ph.D. Thesis, M.I.T. Department of Electrical Engineering and Computer Science.

[13] Parker, L.E. (2008) Distributed Intelligence: Overview of the Field and Its Application in Multi-Robot Systems. Electrical Engineering, 2, 5-14.

[14] Wang, T., Gautero, F., Sabourin, C. and Madani, K. (2011) A Neural Fuzzy Inference Based Adaptive Controller Using Learning Process for Nonholonomic Robots. International Work-conference on Artificial Neural Networks (IWANN 2011), CD-Rom Proceedings, 65-72. http://dx.doi.org/10.1007/978-3-642-21501-8 9

[15] Wang, T., Ramik, D.M., Sabourin, C. and Madani, K. (2012) Intelligent Systems for Industrial Robotics: Application in Logistic Field. Industrial Robot: An International Journal, 39, 251-259. http://dx.doi.org/10.1108/01439911211217071

[16] Wang, T., Ramik, D.M., Sabourin, C. and Madani, K. (2011) Machine-Learning for Heterogeneous Multi-Robots Systems in Logistic Application Frame. Proceedings of the 14th International Conference on Climbing and Walking Robots and the Support Technologies for Mobile Machines (CLAWAR2011), Paris, September 2011, 207-222. http://dx.doi.org/10.1142/9789814374286_0024 